\title{
Characteristics and safety of interventions and procedures performed during catheterisation of patients with congenital heart disease: early report from the national cardiovascular data registry
}

\author{
Ralf Holzer, ${ }^{1}$ Robert Beekman, ${ }^{2}$ Lee Benson, ${ }^{3}$ Lisa Bergersen, ${ }^{4}$ Natalie Jayaram, ${ }^{5}$ Kathy Jenkins, ${ }^{4}$ \\ Kevin Kennedy, ${ }^{6}$ John Moore, ${ }^{7}$ Richard Ringel, ${ }^{8}$ Jonathan Rome, ${ }^{9}$ Robert Vincent, ${ }^{10}$ \\ Gerard R. Martin ${ }^{11}$ \\ ${ }^{1}$ Sidra Medical E Research Center, Doba, Qatar; ${ }^{2}$ Cincinnati Children's Hospital, Cincinnati, United States of America; \\ ${ }^{3}$ Toronto Hospital for Sick Children, Toronto, Canada; ${ }^{4}$ Boston Children's Hospital, Boston; ${ }^{5}$ Saint Luke's Mid America \\ Heart Institute; ${ }^{6}$ Saint Luke's Health System, Kansas City; ${ }^{7}$ Rady Children's Hospital, San Dieho; ${ }^{8} J o b n$ Hopkins \\ Hospital, Baltimore; ${ }^{9}$ Children's Hospital of Philadelpbia, Philadelphia; ${ }^{10}$ Children's Healthcare of Atlanta, Atlanta; \\ ${ }^{11}$ Children's National Medical Center, Washington, United States of America
}

\begin{abstract}
Objectives: The objective of this study was to report procedural characteristics and adverse events on the data collected in the IMproving Paediatric and Adult Congenital Treatment registry. Background: The IMproving Paediatric and Adult Congenital Treatment- registry is a catheterisation registry focussed on paediatric and adult patients with congenital heart disease who are undergoing diagnostic catheterisations and catheter-based interventions. This study reports procedural characteristics and adverse events of patients who have undergone selected catheterisation procedures from January, 2011 to June, 2013. Methods: Demographic, clinical, procedural, and institutional data elements were collected at participating centres and entered via either a web-based platform or software provided by the American College of Cardiology-certified vendors, and were collected in a secure, centralised database. For the purpose of this study, procedures that were not classified as one of the 'core' IMproving Paediatric and Adult Congenital Treatment procedures originally chosen for additional data collection were identified and selected for further data analysis. Results: During the time frame of data collection, a total of 8021 cases were classified as other procedures and/or multiple procedures. The most commonly performed case types - isolated or in combination with other procedures - were right ventricular biopsy in 3433 (42.8\%), conduit/MPA interventions in 979 (12.3\%), and systemic pulmonary artery collateral occlusion in $601(7.5 \%)$. For the whole cohort, adverse events of any severity occurred in $957(12.0 \%)$ cases, whereas major adverse events occurred in $113(1.4 \%)$ cases; six patients $(0.1 \%)$ died in the catheterisation laboratory. Conclusions: The IMproving Paediatric and Adult Congenital Treatment registry has provided important data on the frequency and spectrum of cardiac catheterisation procedures performed in the present era. For many procedures, more data and work are needed to identify more subtle differences between case categories, especially as it relates to the incidence of major adverse events, and to further develop a risk-adjustment methodology to allow equitable comparisons among institutions.
\end{abstract}

Keywords: Cardiac catheterisation; adverse events; congenital heart disease

Received: 22 March 2015; Accepted: 13 September 2015; First published online: 12 October 2015

Correspondence to: Dr R. J. Holzer, MD, MSc, FSCAI, Director Cardiac Catheterization \& Interventional Therapy, Interim Division Chief, Sidra Cardiovascular Center of Excellence, P. O. Box 26999, Doha, Qatar. Tel: +974-4012-5881; E-mail: rholzer@sidra.org 


\section{Background}

A variety of registries such as Congenital Cardiac Catheterization Project on Outcomes, Mid Atlantic Group of Interventional Cardiology, and Congenital Cardiovascular Interventional Study Consortium have reported data on outcomes of congenital cardiac catheterisation. ${ }^{1-4}$ The data were frequently provided for isolated procedures such as patent ductus arteriosus, pulmonary valvuloplasty, pulmonary artery rehabilitation, atrial septal defect closure, as well as for the treatment of coarctation. ${ }^{5-8}$ Other reports focussed on a general description of the frequency of adverse events as well as risk-adjustment

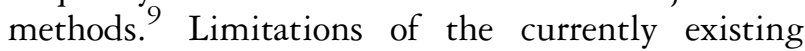
registries include the total number of cases submitted as well as the number of participating centres.

The IMproving Paediatric and Adult Congenital Treatment registry is now operating in over 90 hospitals and has recently reported results on selected "core" procedural categories, which were originally chosen to collect additional procedural details for future assessment of procedure-specific risk and efficacy outcomes. ${ }^{10}$ This original selection of "core" procedures included mainly procedure types that were considered "common" and potentially amenable for the development of additional procedural outcome metrics. The publications on those "core" procedures did not include data on cases that were not captured within these "core" IMproving Paediatric and Adult Congenital Treatment procedural categories; however, these "non-core" cases account for a large number of the procedures that are performed in the catheterisation laboratory, and although many of these cases have no clearly established outcome parameters, providing basic data on these procedures could aid in this process. The purpose of this report was to describe the frequency and basic procedural characteristics of those "non-core" cases, as well as providing data on associated adverse events.

\section{Methods}

\section{General methodology}

The IMproving Paediatric and Adult Congenital Treatment registry is an initiative of the American College of Cardiology Foundation with partnering support from The Society for Cardiovascular Angiography and Interventions and the American Academy of Pediatrics, and it has been previously described by Martin et al. ${ }^{11}$ The registry collects data for use in the development of performance and quality metrics, quality improvement programmes, and peer-reviewed outcomes research focussed on paediatric and adult patients with congenital heart disease who are undergoing diagnostic catheterisations and catheter-based interventions. Demographic, clinical, procedural, and institutional data elements are collected at participating centres and entered via either a web-based platform or a software provided by ACC-certified vendors and are collected in a secure, centralised database. IMproving Paediatric and Adult Congenital Treatment registry has in place a data quality programme consistent with that described for The National Cardiovascular Data Registry. ${ }^{12} \mathrm{~A}$ comprehensive description of IMproving Paediatric and Adult Congenital Treatment Registry data elements and definitions is available at http://www. ncdr.com.

\section{Specific methodology}

The focus of this study was to analyse IMproving Paediatric and Adult Congenital Treatment registry data pertaining to procedural results and outcomes in patients who undergo combined procedures or procedures that were not classified as one of the following IMproving Paediatric and Adult Congenital Treatment 'core' procedures originally chosen for additional data collection: atrial septal defect, device closure of patent ductus arteriosus, pulmonary valvuloplasty, aortic valvuloplasty, coarctation of the aorta angioplasty and stenting, proximal pulmonary artery stenting, and any purely diagnostic cardiac catheterisation. These isolated procedure types had been originally chosen as separate procedural categories within the IMproving Paediatric and Adult Congenital Treatment registry and results on these were previously reported by Moore et al. ${ }^{10}$ For the purpose of this study, the above procedure types are referred to as "core" procedures. In contrast, the subjects of this study, which are all other procedures, captured within the IMproving Paediatric and Adult Congenital Treatment registry are referred to as "non-core" procedures.

Registry data from January, 2011 to June, 2013 were collected from a total of 8021 cardiac catheterisation cases. A single trip to the catheterisation laboratory was defined as a case, whereas the term "episode of care" refers to hospital admission following cardiac catheterisation, up to a maximum of 30 days after the procedure or hospital discharge, whichever occurs earlier.

Basic demographic and clinical data were provided for all the cases in this study. The entire study cohort was then separated into various case types, based on the frequency of cases performed, as well as common case characteristics. Table 1 summarises all the cases collected in this cohort, as well as the number of cases of "core" procedures performed within the same time period. Individual data of "non-core" cases were then separated into groups that had no overlap between 
Table 1. Frequency of procedural categories collected within the IMPACT registry.

\begin{tabular}{|c|c|}
\hline Procedure & Count $(\mathrm{n})$ \\
\hline Proximal PA intervention & $738(3.7 \%)$ \\
\hline Balloon angioplasty only & $238(1.2 \%)$ \\
\hline Stent only** & $320(1.6 \%)$ \\
\hline Balloon angioplasty and stent & $48(0.2 \%)$ \\
\hline Balloon and/or stent with other intervention(s) & $452(2.3 \%)$ \\
\hline Peripheral PA intervention & $451(2.3 \%)$ \\
\hline Balloon angioplasty only & $160(0.8 \%)$ \\
\hline Stent only & $32(0.2 \%)$ \\
\hline Balloon angioplasty and stent & $20(0.1 \%)$ \\
\hline Balloon and/or stent with other intervention(s) & $239(1.2 \%)$ \\
\hline Conduit/MPA intervention & $979(5.0 \%)$ \\
\hline Balloon angioplasty only & $399(2.0 \%)$ \\
\hline Stent only & $78(0.4 \%)$ \\
\hline Balloon angioplasty and stent & $40(0.2 \%)$ \\
\hline Balloon and/or stent with other intervention(s) & $462(2.2 \%)$ \\
\hline $\begin{array}{l}\text { Multi-level PA intervention (balloon and/or stent in at } \\
\text { least two PA locations: proximal, peripheral, conduit/MPA) }\end{array}$ & $331(1.7 \%)$ \\
\hline Balloon angioplasty and/or stent & $267(1.4 \%)$ \\
\hline Balloon and/or stent with other intervention(s) & $64(0.3 \%)$ \\
\hline Caval vein intervention & $236(1.2 \%)$ \\
\hline Balloon angioplasty only & $72(0.4 \%)$ \\
\hline Stent only & $41(0.2 \%)$ \\
\hline Balloon angioplasty and stent & $19(0.1 \%)$ \\
\hline Balloon and/or stent with other intervention(s) & $104(0.5 \%)$ \\
\hline Pulmonary vein intervention & $202(1.0 \%)$ \\
\hline Balloon angioplasty only & $102(0.5 \%)$ \\
\hline Stent only & $17(0.1 \%)$ \\
\hline Balloon angioplasty and stent & $21(0.1 \%)$ \\
\hline Balloon angioplasty and/or stent + ASD intervention & $22(0.1 \%)$ \\
\hline Balloon and/or stent with other intervention(s) & $40(0.2 \%)$ \\
\hline Aorta & $800(4.0 \%)$ \\
\hline Balloon angioplasty or stent** & $671(3.4 \%)$ \\
\hline Balloon angioplasty or stent with other intervention(s) & $129(0.7 \%)$ \\
\hline Other artery intervention & $87(0.4 \%)$ \\
\hline Balloon angioplasty and/or stent & $49(0.2 \%)$ \\
\hline Balloon and/or stent with other intervention(s) & $38(0.2 \%)$ \\
\hline Other vein intervention & $100(0.5 \%)$ \\
\hline Balloon angioplasty and/or stent & $30(0.2 \%)$ \\
\hline Balloon and/or stent with other intervention(s) & $70(0.4 \%)$ \\
\hline Sano (conduit) or shunt intervention & $141(0.7 \%)$ \\
\hline Balloon angioplasty and/or stent & $89(0.5 \%)$ \\
\hline Balloon and/or stent with other intervention(s) & $52(0.3 \%)$ \\
\hline PDA intervention & $105(0.5 \%)$ \\
\hline Balloon angioplasty and/or stent & $90(0.5 \%)$ \\
\hline Balloon and/or stent with other intervention(s) & $15(0.1 \%)$ \\
\hline Fontan or atrial baffle intervention & $50(0.3 \%)$ \\
\hline Balloon angioplasty and/or stent & $27(0.1 \%)$ \\
\hline Balloon and/or stent with other intervention(s) & $23(0.1 \%)$ \\
\hline Fontan fenestration intervention & $36(0.2 \%)$ \\
\hline Balloon angioplasty and/or stent & $18(0.1 \%)$ \\
\hline Balloon and/or stent with other intervention(s) & $18(0.1 \%)$ \\
\hline ASD occlusion & $1434(7.3 \%)$ \\
\hline Isolated $* *$ & $1362(6.9 \%)$ \\
\hline With other intervention(s) & $72(0.4 \%)$ \\
\hline PFO occlusion & $203(1.0 \%)$ \\
\hline Isolated & $171(0.9 \%)$ \\
\hline With other intervention & $32(0.2 \%)$ \\
\hline Fenestration or baffle leak occlusion & $160(0.8 \%)$ \\
\hline Isolated & $95(0.5 \%)$ \\
\hline With other intervention & $65(0.3 \%)$ \\
\hline PDA occlusion & $1426(7.2 \%)$ \\
\hline Isolated $* *$ & $1375(7.0 \%)$ \\
\hline With other intervention(s) & $51(0.3 \%)$ \\
\hline
\end{tabular}


Table 1. Continued

\begin{tabular}{|c|c|}
\hline Procedure & Count $(n)$ \\
\hline VSD occlusion & $65(0.3 \%)$ \\
\hline Isolated & $44(0.2 \%)$ \\
\hline With other intervention & $21(0.1 \%)$ \\
\hline Systemic vein to PV collateral occlusion & $253(1.3 \%)$ \\
\hline Isolated & $133(0.7 \%)$ \\
\hline With other intervention & $120(0.6 \%)$ \\
\hline Systemic artery to PA collateral occlusion & $601(3.0 \%)$ \\
\hline Isolated & $383(1.9 \%)$ \\
\hline With other intervention & $218(1.1 \%)$ \\
\hline Other vascular structure device or coil placement & $283(1.4 \%)$ \\
\hline Isolated & $184(0.9 \%)$ \\
\hline With other intervention & $99(0.5 \%)$ \\
\hline Aortic valvuloplasty & $357(1.8 \%)$ \\
\hline Isolated $* *$ & $340(1.7 \%)$ \\
\hline With other intervention(s) & $17(0.1 \%)$ \\
\hline Pulmonary valvuloplasty & $768(3.9 \%)$ \\
\hline Isolated $* *$ & $661(3.3 \%)$ \\
\hline With other intervention(s) & $107(0.5 \%)$ \\
\hline Mitral valvuloplasty & $30(0.2 \%)$ \\
\hline Isolated & $13(0.1 \%)$ \\
\hline With atrial septal intervention & $11(0.1 \%)$ \\
\hline With other intervention & $6(<0.05 \%)$ \\
\hline Tricuspid valvuloplasty & $12(0.1 \%)$ \\
\hline Isolated & $5(<0.05 \%)$ \\
\hline With other intervention & $7(<0.05 \%)$ \\
\hline Transcatheter pulmonary valve implantation & $374(1.9 \%)$ \\
\hline Isolated & $158(0.8 \%)$ \\
\hline With balloon and/or stent of MPA/conduit & $95(0.5 \%)$ \\
\hline With other intervention & $121(0.6 \%)$ \\
\hline Balloon atrial septostomy & $274(1.4 \%)$ \\
\hline Isolated & $241(1.2 \%)$ \\
\hline With other intervention & $33(0.2 \%)$ \\
\hline Atrial septal intervention (non-BAS) & $221(1.1 \%)$ \\
\hline Isolated & $111(0.6 \%)$ \\
\hline With other intervention & $110(0.6 \%)$ \\
\hline RV biopsy & $3433(17.4 \%)$ \\
\hline Isolated & $3372(17.1 \%)$ \\
\hline With other intervention & $61(0.3 \%)$ \\
\hline Vascular/valvar perforation & $35(0.2 \%)$ \\
\hline Isolated & $12(0.1 \%)$ \\
\hline With other intervention & $23(0.1 \%)$ \\
\hline Diagnostic only catheterisation** & $7010(35.5 \%)$ \\
\hline
\end{tabular}

ASD $=$ atrial septal defect; BAS $=$ balloon atrial septostomy; IMPACT $=$ IMproving Paediatric and Adult Congenital Treatment; MPA = main pulmonary artery; $\mathrm{PA}=$ pulmonary artery; $\mathrm{PDA}=$ patent ductus arteriosus; $\mathrm{PFO}=$ patent foramen ovale; $\mathrm{RV}=$ right ventricle; $\mathrm{VSD}=$ ventricular septal defect

Case types marked with ** were "core" IMPACT case types and are not further analysed in detail within this report. This includes isolated cases of the following categories: diagnostic catheterisation, balloon pulmonary valvuloplasty, balloon aortic valvuloplasty, PDA occlusion, ASD occlusion, balloon angioplasty or stenting of coarctation, and proximal PA stenting. Percentages refer to the total number of cases in the registry $(\mathrm{n}=19,760=100 \%)$ and a case can be listed in more than one procedural category

individual case types (Table 2). Data collected for these individual case types included weight, case time, contrast administered, as well as data on adverse events and death (Fig 1).

Adverse events captured within the IMproving Paediatric and Adult Congenital Treatment registry are listed in Table 3. Major adverse events are defined as the need for permanent or temporary pacemaker, cardiac arrest, cardiac tamponade requiring pericardial drainage, embolic stroke, as well as unplanned cardiac surgery due to a catheter-related adverse event. Bleeding events after catheterisation are captured if they are associated with either a drop in haemoglobin of $\geqslant 3 \mathrm{mg} / \mathrm{dl}$, or a transfusion of whole blood or packed red 
Table 2. Case categories without overlap.

\begin{tabular}{|c|c|}
\hline \multicolumn{2}{|l|}{ Proximal PA } \\
\hline Balloon & $238(3.0 \%)$ \\
\hline Balloon and stent & $48(0.6 \%)$ \\
\hline \multicolumn{2}{|l|}{ Peripheral PA } \\
\hline Balloon & $160(2.0 \%)$ \\
\hline Stent & $32(0.4 \%)$ \\
\hline Balloon and stent & $20(0.2 \%)$ \\
\hline \multicolumn{2}{|l|}{ Conduit/MPA } \\
\hline Balloon & $399(5.0 \%)$ \\
\hline Stent & $78(1.0 \%)$ \\
\hline Balloon and Stent & $40(0.5 \%)$ \\
\hline Multi-level PA - balloon and/or stent & $267(3.3 \%)$ \\
\hline \multicolumn{2}{|l|}{ Caval vein } \\
\hline Balloon & $72(0.9 \%)$ \\
\hline Stent & $41(0.5 \%)$ \\
\hline Balloon and stent & $19(0.2 \%)$ \\
\hline \multicolumn{2}{|l|}{ Pulmonary vein } \\
\hline Balloon & $102(1.3 \%)$ \\
\hline Stent & $17(0.2 \%)$ \\
\hline Balloon and stent & $21(0.3 \%)$ \\
\hline Other artery and/or vein - balloon and/or stent & $79(1 \%)$ \\
\hline Sano (conduit), shunt, and/or PDA - balloon and/or stent & $179(2.2 \%)$ \\
\hline Fontan and/or baffle - balloon and/or stent & $27(0.3 \%)$ \\
\hline Fontan fenestration - balloon and/or stent & $18(0.2 \%)$ \\
\hline PFO occlusion & $171(2.1 \%)$ \\
\hline Fenestration and/or baffle leak occlusion & $95(1.2 \%)$ \\
\hline VSD occlusion & $44(0.5 \%)$ \\
\hline Systemic vein to PV and/or systemic artery to PA collateral occlusion & $546(6.8 \%)$ \\
\hline Other vascular structure device or coil placement & $184(2.3 \%)$ \\
\hline Mitral and/or tricuspid valvuloplasty & $18(0.2 \%)$ \\
\hline \multicolumn{2}{|l|}{ Transcatheter pulmonary valve } \\
\hline Isolated & $158(2 \%)$ \\
\hline With balloon and/or stent of MPA/conduit & $138(1.7 \%)$ \\
\hline Balloon atrial septostomy & $241(3 \%)$ \\
\hline Atrial septal intervention ( \pm BAS) & $129(1.6 \%)$ \\
\hline RV biopsy & $3372(42.0 \%)$ \\
\hline Vascular/valvar perforation & $12(0.1 \%)$ \\
\hline Other isolated or combined procedures & $1056(13.2 \%)$ \\
\hline
\end{tabular}

$\mathrm{BAS}=$ balloon atrial septostomy; $\mathrm{MPA}=$ main pulmonary artery; $\mathrm{PA}=$ pulmonary artery; $\mathrm{PDA}=$ patent ductus arteriosus; $\mathrm{PFO}=$ patent foramen ovale; $\mathrm{PV}=$ pulmonary vein; $\mathrm{RV}=$ right ventricle; $\mathrm{VSD}=$ ventricular septal defect Any case is only listed in one of the case categories. Percentages refer to the total number of 'other' or 'combined' procedures $(\mathrm{n}=8021=100 \%)$

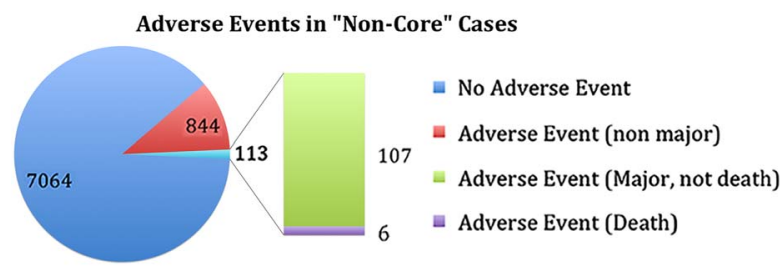

Figure 1.

Adverse events encountered in "non-core" cases.

blood cells, or any procedural intervention/surgery at the bleeding site to reverse/stop or correct the bleeding. Adverse events were reported if they occured at any time from cardiac catheterisation until 30 days after the procedure or hospital discharge, whichever occured earlier (episode of care), except for an embolic stroke, which was reported up to 72 hours after the procedure. If more than one catheterisation procedure was performed during the same episode of care, adverse events were captured until the subsequent cardiac catheterisation, except for bleeding and transfusion events, which are captured only up to 72 hours after the procedure. Death was not included as part of the adverse events, but reported separately as either death in the catheterisation laboratory or death within the same episode of care as defined above.

\section{Inclusion and exclusion criteria}

Inclusion criteria:

- Any case submitted to the IMproving Paediatric and Adult Congenital Treatment registry 
Table 3. Adverse events captured within the IMPACT registry.

\begin{tabular}{ll}
\hline Any adverse event & Major adverse event \\
\hline Cardiac arrest & $\begin{array}{l}\text { Need for permanent or temporary pacemaker } \\
\text { Cardiac arrest }\end{array}$ \\
$\begin{array}{l}\text { Air embolus } \\
\text { Arrhythmia }\end{array}$ & $\begin{array}{l}\text { Cardiac tamponade (requiring pericardial drainage) } \\
\text { Arrhythmia requiring cardioversion }\end{array}$ \\
Antiarrhythmic medication & Unplanned cardiac surgery (due to catheterisation AE) \\
Permanent or temporary pacemaker & \\
New heart valve regurgitation & \\
Tamponade & \\
Embolic stroke & \\
New requirement for dialysis & \\
Airway event requiring intubation & \\
Event requiring ECMO or LVAD* & \\
Bleeding event & \\
Bleeding event at the access site & \\
Haematoma at access site & \\
Retroperitoneal & \\
Gastrointestinal or genital-urinary bleeding & \\
Red blood cell/whole blood transfusion & \\
Device malposition & \\
Device thrombus & \\
Device embolisation & \\
Unplanned surgery (due to catheterisation AE) & \\
Subsequent cardiac catheterisation & \\
(due to catheterisation AE) & \\
\hline AE = adverse events; ECMO = extracorporeal membrane oxygenation; IMPACT = IMproving Paediatric and Adult \\
Congenital Treatment; LVAD = left ventricular assist device \\
$*$ Events requiring ECMO or LVAD will be classified as a "Major adverse event" in the next version of the IMPACT \\
registry (version 2)
\end{tabular}

Exclusion criteria:

- Any case that only included one of the "core" interventional procedures, as defined in the specific methodlogy section

- Any procedure that was solely diagnostic

\section{Statistics}

Basic descriptive data were calculated for all cardiac catheterisation cases and case types meeting the inclusion and exclusion criteria. Continuous variables are presented as median and interquartile range. Categorical variables are presented as frequency, using the number of cases for which the data element was provided as $100 \%$.

\section{Results}

\section{Basic demographic and clinical data}

Within the IMproving Paediatric and Adult Congenital Treatment registry, a total of 8021 cases were classified as other procedures and/or multiple procedures, performed during 7691 episodes of care. These "non-core" cases formed the study cohort. The median weight was $23.1 \mathrm{~kg}$ (interquartile range 10,
$9,53.8)$. The age was $<30$ days for $451(5.6 \%)$, equal to 30 days and below or equal to 1 year for 1017 $(12.7 \%)$, above 1 year and below or equal to 18 years for $5210(65.0 \%)$, and above 18 years for 1343 $(16.7 \%)$ of the cases. A history of previous cardiac catheterisation was present in 6507 (82.4\%) cases, and $619(9.5 \%)$ cases underwent cardiac catheterisation within the preceding 30 days. A history of previous cardiac surgery was present in $6220(78 \%)$ cases, and $996(16 \%)$ cases had cardiac surgery within the preceding 30 days. Single ventricle anatomy was present in 1655 $(20.7 \%)$ cases.

The majority of cases were performed electively $(6738,84.3 \%)$, followed by urgent $(962,12.0 \%)$, emergent $(259,3.2 \%)$, and salvage $(32,0.4 \%)$ procedures. General anaesthesia was used in 6202 (77.5\%) cases, with elective intubation at the start in $4935(61.5 \%)$ cases. The median case time was 80 minutes (interquartile range 39,138 ), and the median amount of contrast administered was $2.1 \mathrm{ml} / \mathrm{kg}$ (interquartile range $0.3,4.5$ ). A systemic ventricular end-diastolic pressure equal to or above $18 \mathrm{mmHg}$ was present in $352(8.2 \%)$, and a cardiac index equal to or below 2.8 was present in 1663 $(25.8 \%)$ of the cases. 


\section{Individual procedural data}

A summary of all cases performed is listed in Tables 1 and 2. The most commonly performed case - in isolation or in combination with other procedures was right ventricular biopsy, accounting for 3433 (42.8\%) of all "non-core" cases within the IMproving Paediatric and Adult Congenital Treatment registry. Pulmonary artery interventions - in isolation or in combination with other procedures - were performed proximally in 738 (9.2\%), peripherally in 451 $(5.6 \%)$, and at the conduit/main pulmonary artery in $979(12.2 \%)$ cases. Transcatheter pulmonary valve implantation was carried out in $374(4.7 \%)$ cases. Aortopulmonary collaterals were occluded in 601 (7.5\%), and venous collaterals were occluded in 253 $(3.2 \%)$ of the cases.

Isolated case types are listed in Table 4. The longest median case times were seen for combined balloon angioplasty and stent placement in the peripheral pulmonary arteries (178 minutes) as well as the pulmonary veins (180 minutes), whereas the shortest median case times were seen for right ventricular biopsy (38 minutes) and patent foramen ovale occlusion (60 minutes). The only other isolated case type with a case time of $<100$ minutes was balloon angioplasty and/or stent placement within a Sano conduit, Blalock-Taussig shunt, or patent arterial duct (94 minutes).

Among all, four case types had an associated median use of contrast exceeding $5 \mathrm{ml} / \mathrm{kg}$. These included multi-level pulmonary artery rehabilitation with balloon angioplasty and/or stent placement $(5.0 \mathrm{ml} / \mathrm{kg})$, balloon angioplasty and stent placement in the peripheral pulmonary arteries $(5.7 \mathrm{ml} / \mathrm{kg})$ or at the right ventricle-to-pulmonary artery conduit/main pulmonary artery $(5.8 \mathrm{ml} / \mathrm{kg})$, as well as vascular/ valvar perforation cases $(5.9 \mathrm{ml} / \mathrm{kg})$.

\section{Adverse events}

Data on adverse events for individual case types are listed in Table 4. For the entire cohort, adverse events of any severity occurred in $957(12.0 \%)$ cases, whereas major adverse events occurred in $113(1.4 \%)$ cases (Fig 1); six patients $(0.1 \%)$ died in the catheterisation laboratory, and 187 patients died within the same episode of care of causes not necessarily related to cardiac catheterisation. Cardiac arrest occurred in 76 $(1.0 \%)$, cardiac tamponade in $15(0.2 \%)$, embolic stroke in $5(0.1 \%)$, device malposition requiring surgery in $10(0.1 \%)$, device embolisation requiring retrieval in $30(0.4 \%)$, new requirement for dialysis in $11(0.1 \%)$, need for extra-corporal membrane oxygenation in $28(0.4 \%)$, and an airway event requiring intubation in $59(0.7 \%)$ cases. Bleeding events occurred in $140(1.8 \%)$ cases, with $69(49.6 \%)$ cases bleeding at the access site and $44(31.7 \%)$ with a haematoma at the access site. Blood transfusion was administered in $387(4.8 \%)$ cases. Arrhythmias occurred in $186(2.3 \%)$, requiring cardioversion in 55 (29.6\%), antiarrhythmic medication in 87 (46.8\%), and temporary pacing in $16(8.6 \%)$ of the cases, no permanent pacemaker was required. Secondary to a catheterisation complication, unplanned cardiac surgery was required in $25(0.3 \%)$ and unplanned vascular surgery in $11(0.1 \%)$ cases. Unplanned other surgery was required in $30(0.4 \%)$ cases, $10.0 \%$ of which were secondary to catheter-related complications. Red blood cell transfusion was required in 387 $(4.8 \%)$ cases.

For individual cases, the number of major adverse events was generally very low, and therefore difficult to compare, with only five case categories having more than three cases with a major adverse event. These were balloon angioplasty and/or stent placement in the Sano conduit, shunt, or patent arterial duct $(\mathrm{n}=5,2.8 \%)$, balloon atrial septostomy $(\mathrm{n}=13,5.5 \%)$, as well as other atrial septal interventions with or without balloon atrial septostomy $(\mathrm{n}=10,7.8 \%)$.

There were four case types where the incidence of cases with any adverse event was $>30 \%$, which included balloon angioplasty and stent placement in the peripheral pulmonary arteries $(9 / 20,45.0 \%)$, balloon angioplasty and stent placement in the pulmonary veins $(7 / 21,33.3 \%)$, balloon angioplasty and/or stent placement in the Sano conduit, shunt, or patent arterial duct $(66 / 179,37.5 \%)$, as well as vascular/valvar perforation $(5 / 12,41.7 \%)$.

In total, five case categories had $<10 \%$ of cases with any type of adverse events. These included balloon angioplasty of the caval vein $(6 / 72,8.3 \%)$, patent foramen ovale occlusion $(3 / 171,1.8 \%)$, occlusion of a fenestration or baffle leak $(6 / 95,6.3 \%)$, right ventricular biopsy $(108 / 3372,3.2 \%)$, as well as mitral or tricuspid valvuloplasty $(1 / 18,5.6 \%)$.

\section{Discussion}

This report provides an overview of the types of "non-core" cases submitted to the IMproving Paediatric and Adult Congenital Treatment registry, excluding the "core" IMproving Paediatric and Adult Congenital Treatment procedure types that are reported separately. ${ }^{10}$ Although a variety of registries have published data on individual case types as well as adverse events, beyond the results of the Congenital Cardiac Catheterization Project on Outcomes registry there are limited multi-centre data available on the frequency of the various case types that are performed in the cardiac catheterisation laboratory in the present era. 
Table 4. Basic characteristics of individual case types within the IMPACT registry.

\begin{tabular}{|c|c|c|c|}
\hline & \multicolumn{2}{|l|}{ Proximal PA } & \multirow{2}{*}{$\frac{\text { Multi-level PA }}{\text { Balloon and/or stent }}$} \\
\hline & Balloon & Balloon and stent & \\
\hline Number of cases & 238 & 48 & 267 \\
\hline Weight & $12.7[8.2,23.1]$ & $15.9[9.8,34.0]$ & $16.6[10.0,29.8]$ \\
\hline Procedure time & $111.0[82.0,146.0]$ & $149.0[98.5,178.5]$ & $148.5[117.0,212.0]$ \\
\hline Contrast & $3.9[2.5,5.4]$ & $3.7[3.0,5.9]$ & $5.0[3.5,6.6]$ \\
\hline Any adverse event & $31(13.0 \%)$ & $5(10.4 \%)$ & $53(20.1 \%)$ \\
\hline Major adverse event & $2(0.8 \%)$ & $0(0.0 \%)$ & $3(1.1 \%)$ \\
\hline Death in the catheterisation lab & $2(0.8 \%)$ & $0(0.0 \%)$ & $0(0.0 \%)$ \\
\hline
\end{tabular}

Peripheral PA

\begin{tabular}{lccc} 
& Balloon & Stent & Balloon and stent \\
\cline { 2 - 4 } Number of cases & 160 & 32 & 20 \\
Weight & $13.6[9.0,27.6]$ & $13.7[9.2,24.7]$ & $13.0[10.4,25.4]$ \\
Procedure time & $137.0[100.0,194.0]$ & $147.0[101.5,192.0]$ & $178.0[145.0,267.0]$ \\
Contrast & $4.3[0.0,11.5]$ & $4.9[3.3,6.0]$ & $5.7[3.3,7.4]$ \\
Any adverse event & $22(13.8 \%)$ & $7(21.9 \%)$ & $9(45.0 \%)$ \\
Major adverse event & $0(0.0 \%)$ & $2(6.3 \%)$ & $0(0.0 \%)$ \\
Death in the catheterisation lab & $0(0.0 \%)$ & $0(0.0 \%)$ & $0(0.0 \%)$
\end{tabular}

Conduit/MPA

\begin{tabular}{llll} 
& Conduit/MPA & & \\
\cline { 2 - 4 } & Balloon & Stent & Balloon and stent \\
\hline Number of cases & 399 & 78 & 40 \\
Weight & $16.7[8.5,43.1]$ & $14.7[7.6,34.1]$ & $13.8[8.3,26.9]$ \\
Procedure time & $117.0[89.0,151.5]$ & $123.0[92.0,154.0]$ & $159.0[115.0,208.0]$ \\
Contrast & $3.9[2.6,5.4]$ & $4.5[3.4,6.2]$ & $5.8[4.4,8.3]$ \\
Any adverse event & $49(12.3 \%)$ & $13(16.7 \%)$ & $11(28.2 \%)$ \\
Major adverse event & $3(0.8 \%)$ & $3(3.8 \%)$ & $1(2.6 \%)$ \\
Death in the catheterisation lab & $0(0.0 \%)$ & $0(0.0 \%)$ & $0(0.0 \%)$
\end{tabular}

\begin{tabular}{|c|c|c|c|c|}
\hline & \multicolumn{3}{|l|}{ Caval vein } & \\
\hline & Balloon & Stent & Balloon and stent & \\
\hline Number of cases & 72 & 41 & 19 & \\
\hline Weight & $16.9[10.9,56.4]$ & $21.5[10.3,70.5]$ & $24.3[12.2,62.6]$ & \\
\hline Procedure time & $116.0[75.0,148.0]$ & $127.0[115.0,175.0]$ & $131.0[97.0,230.0]$ & \\
\hline Contrast & $3.2[1.8,4.3]$ & $2.9[1.8,3.8]$ & $2.8[1.7,4.4]$ & \\
\hline Any adverse event & $6(8.3 \%)$ & $8(19.5 \%)$ & $4(21.1 \%)$ & \\
\hline Major adverse event & $1(1.4 \%)$ & $1(2.4 \%)$ & $0(0.0 \%)$ & \\
\hline \multirow[t]{3}{*}{ Death in the catheterisation lab } & $0(0.0 \%)$ & $0(0.0 \%)$ & $0(0.0 \%)$ & \\
\hline & \multicolumn{3}{|l|}{ Pulmonary vein } & \\
\hline & Balloon & Stent & Balloon and stent & \\
\hline Number of cases & 102 & 17 & 21 & \\
\hline Weight & $9.5[6.4,13.9]$ & $11.7[4.2,59.9]$ & $9.8[5.8,16.4]$ & \\
\hline Procedure time & $140.0[105.0,180.0]$ & $119.0[104.0,211.0]$ & $180.0[118.0,290.0]$ & \\
\hline Contrast & $3.1[2.1,4.7]$ & $2.8[1.4,4.1]$ & $4.5[3.0,5.9]$ & \\
\hline Any adverse event & $25(25.3 \%)$ & $5(29.4 \%)$ & $7(33.3 \%)$ & \\
\hline Major adverse event & $3(3.0 \%)$ & $1(5.9 \%)$ & $1(4.8 \%)$ & \\
\hline \multirow[t]{3}{*}{ Death in the catheterisation lab } & $0(0.0 \%)$ & $0(0.0 \%)$ & $0(0.0 \%)$ & \\
\hline & \multicolumn{4}{|l|}{ Balloon and/or stent } \\
\hline & Other artery/vein & Sano/shunt/PDA & Fontan/baffle & Fontan fenestration \\
\hline Number of cases & 79 & 179 & 27 & 18 \\
\hline Weight & $20.0[4.7,52.5]$ & $3.5[3.0,4.9]$ & $55.7[20.5,71.0]$ & $18.1[14.7,40.5]$ \\
\hline Procedure time & $118.0[72.0,174.0]$ & $94.0[53.0,144.0]$ & $129.0[95.0,175.0]$ & $139.0[98.0,198.0]$ \\
\hline Contrast & $3.3[1.9,5.0]$ & $4.9[3.3,7.0]$ & $2.4[1.5,3.5]$ & $3.5[2.6,4.8]$ \\
\hline Any AE & $22(27.8 \%)$ & $66(37.5 \%)$ & $4(14.8 \%)$ & $3(17.6 \%)$ \\
\hline Major AE & $1(1.3 \%)$ & $5(2.8 \%)$ & $0(0.0 \%)$ & $0(0.0 \%)$ \\
\hline Death in the catheterisation lab & $1(1.3 \%)$ & $1(0.6 \%)$ & $0(0.0 \%)$ & $0(0.0 \%)$ \\
\hline
\end{tabular}




\begin{tabular}{|c|c|c|c|c|}
\hline & \multicolumn{4}{|l|}{ Occlusion } \\
\hline & $\mathrm{PFO}$ & Fenestration/baffle leak & VSD & $\begin{array}{l}\text { Collateral } \\
\text { (vein/artery) }\end{array}$ \\
\hline Number of cases & 171 & 95 & 44 & 546 \\
\hline Weight & $79.0[62.5,92.0]$ & $19.6[15.5,34.4]$ & $12.9[7.2,24.2]$ & $13.7[10.2,25.6]$ \\
\hline Procedure time & $60.0[45.0,78.0]$ & $102.0[75.0,128.0]$ & $140.0[112.5,176.0]$ & $126.5[94.0,173.0]$ \\
\hline Contrast & $0.1[0.0,0.7]$ & $3.9[2.2,5.2]$ & $3.8[2.6,5.3]$ & $4.3[3.0,5.8]$ \\
\hline Any AE & $3(1.8 \%)$ & $6(6.3 \%)$ & $12(27.3 \%)$ & $78(14.4 \%)$ \\
\hline Major AE & $0(0.0 \%)$ & $0(0.0 \%)$ & $1(2.3 \%)$ & $6(1.1 \%)$ \\
\hline \multirow[t]{3}{*}{ Death in the catheterisation lab } & $0(0.0 \%)$ & $0(0.0 \%)$ & $0(0.0 \%)$ & $0(0.0 \%)$ \\
\hline & \multicolumn{4}{|c|}{ Transcatheter pulmonary valve } \\
\hline & \multicolumn{2}{|l|}{ Isolated } & \multicolumn{2}{|l|}{$\begin{array}{l}\text { With balloon/stent of } \\
\text { MPA/conduit }\end{array}$} \\
\hline \multirow{9}{*}{$\begin{array}{l}\text { Number of cases } \\
\text { Weight } \\
\text { Procedure time } \\
\text { Contrast } \\
\text { Any adverse event } \\
\text { Major adverse event } \\
\text { Death in the catheterisation lab }\end{array}$} & \multirow{7}{*}{\multicolumn{2}{|c|}{$\begin{array}{l}158 \\
61.5[50.3,79.4] \\
168.0[127.0,212.0] \\
3.1[2.0,4.1] \\
20(12.7 \%) \\
3(1.9 \%) \\
0(0.0 \%)\end{array}$}} & \multicolumn{2}{|l|}{138} \\
\hline & & & \multicolumn{2}{|l|}{$62.5[44.0,73.5]$} \\
\hline & & & \multicolumn{2}{|l|}{$165.0[125.0,217.0]$} \\
\hline & & & \multicolumn{2}{|l|}{$3.4[2.4,5.2]$} \\
\hline & & & \multicolumn{2}{|l|}{$32(23.2 \%)$} \\
\hline & & & $3(2.2 \%)$ & \\
\hline & & & \multicolumn{2}{|l|}{$0(0.0 \%)$} \\
\hline & \multicolumn{4}{|l|}{ Atrial septal intervention* } \\
\hline & \multicolumn{2}{|l|}{ BAS } & \multicolumn{2}{|l|}{ Other $( \pm \mathrm{BAS})$} \\
\hline \multirow{9}{*}{$\begin{array}{l}\text { Number of cases } \\
\text { Weight } \\
\text { Procedure time } \\
\text { Contrast } \\
\text { Any adverse event } \\
\text { Major adverse event } \\
\text { Death in the catheterisation lab }\end{array}$} & \multicolumn{2}{|l|}{241} & \multicolumn{2}{|l|}{129} \\
\hline & \multicolumn{2}{|l|}{$3.4[2.9,3.8]$} & \multicolumn{2}{|l|}{$5.5[3.5,13.8]$} \\
\hline & \multicolumn{2}{|l|}{$47.0[27.0,88.0]$} & \multicolumn{2}{|l|}{$96.0[68.0,145.0]$} \\
\hline & \multicolumn{2}{|l|}{$1.6[0.0,3.7]$} & \multicolumn{2}{|l|}{$2.8[1.0,4.9]$} \\
\hline & \multicolumn{2}{|l|}{$59(24.8 \%)$} & \multicolumn{2}{|l|}{$38(29.7 \%)$} \\
\hline & \multirow{2}{*}{\multicolumn{2}{|c|}{$\begin{array}{r}13(5.5 \%) \\
1(0.4 \%)\end{array}$}} & \multirow{2}{*}{\multicolumn{2}{|c|}{$\begin{array}{r}10(7.8 \%) \\
0(0.0 \%)\end{array}$}} \\
\hline & & & & \\
\hline & Other procedures & & & \\
\hline & Other vascular device/coil & Mitral/tricuspid valvuloplasty & Vascular/valvar perforations & RV biopsy \\
\hline Number of cases & 184 & 18 & 12 & 3372 \\
\hline Weight & $17.4[9.7,51.0]$ & $60.5[30.0,73.0]$ & $3.7[2.7,4.0]$ & $38.2[18.6,58.5]$ \\
\hline Procedure time & $139.0[92.5,186.0]$ & $126.0[97.0,173.0]$ & $112.5[91.5,136.5]$ & $38.0[22.0,60.0]$ \\
\hline Contrast & $3.9[2.7,5.1]$ & $0.9[0.5,1.2]$ & $5.9[4.6,6.5]$ & $0.2[0.0,0.7]$ \\
\hline Any AE & $19(10.4 \%)$ & $1(5.6 \%)$ & $5(41.7 \%)$ & $108(3.2 \%)$ \\
\hline Major AE & $1(0.5 \%)$ & $1(5.6 \%)$ & $2(16.7 \%)$ & $20(0.6 \%)$ \\
\hline Death in the catheterisation lab & $1(0.5 \%)$ & $0(0.0 \%)$ & $0(0.0 \%)$ & $0(0.0 \%)$ \\
\hline
\end{tabular}

$\mathrm{AE}=$ adverse events; $\mathrm{BAS}=$ balloon atrial septostomy; IMPACT $=$ IMproving Paediatric and Adult Congenital Treatment; MPA = main pulmonary artery; $\mathrm{PA}=$ pulmonary artery; $\mathrm{PDA}=$ patent ductus arteriosus; $\mathrm{RV}=$ right ventricle; $\mathrm{VSD}=$ ventricular septal defect

*The listed atrial septal interventions (of any type) include all the patients with all underlying diagnoses, whether transposition of the great arteries, hypoplastic left heart syndrome, or any other diagnosis

In this data set, the most commonly performed cases included many well-established case types such as pulmonary artery rehabilitation, right ventricular biopsy, collateral occlusion, as well as atrial septal interventions; however, it also included newer case types such as transcatheter pulmonary valve implantation, which was performed in almost $5 \%$ of "non-core" cases submitted to the IMproving Paediatric and Adult Congenital Treatment registry.

The incidence of any adverse events within the IMproving Paediatric and Adult Congenital Treatment registry among "non-core" cases was $12 \%$, whereas major adverse events occurred in $1.4 \%$ of the cases. This lies within the range of adverse events reported for "core" interventional procedures, which ranged from 5.3 to $24.3 \%$ for any adverse events and from 0 to $3.3 \%$ for major adverse events, as reported by Moore et al. ${ }^{10}$ It is important to note that the three of four case categories with the highest incidence of adverse events were among those least frequently performed ( $n<25$ cases), whereas four of the five case categories with the lowest incidence of adverse events were among those more frequently performed case categories ( $\mathrm{n}>70$ cases). 
For diagnostic cases captured in the IMproving Paediatric and Adult Congenital Treatment registry, any adverse events occurred in $10 \%$ of the cases, whereas major adverse events occurred in $1.4 \%$ of the cases, as reported by Vincent et al. ${ }^{13}$ Given the very wide variability of case types captured within the group of "non-core" procedures, it is impossible to derive any more detailed conclusions from this data, however.

In addition to IMproving Paediatric and Adult Congenital Treatment data, the only other recent multi-centre registry data available on catheterisation-related adverse events originate from the Congenital Cardiac Catheterization Project on Outcomes registry. ${ }^{1}$ It reported an incidence of any adverse event of $20 \%$ for interventional cases, with 9\% being high-severity adverse events; however, the definition of adverse events was broad and not limited to just a few selected types of adverse events, as is the case with the IMproving Paediatric and Adult Congenital Treatment registry. Importantly, however, the incidence of life-threatening adverse events reported from the Congenital Cardiac Catheterization Project on Outcomes registry (2.1\%) was more similar to the incidence of major adverse events of $1.4 \%$ reported in the present study. ${ }^{14}$

Although the incidence of adverse events is difficult to compare between these two registries, all four case types of the IMproving Paediatric and Adult Congenital Treatment registry that had associated adverse events in $>30 \%$ of cases had previously also been identified as high-risk "procedure type risk groups" by the data derived from the Congenital Cardiac Catheterization Project on Outcomes registry. ${ }^{15}$ Further work has been completed on the IMproving Paediatric and Adult Congenital Treatment data set to develop a risk stratification and risk-adjustment method, and to compare this with the catheterisation for congenital heart disease adjustment for risk method derived from the Congenital Cardiac Catheterization Project on Outcomes data set, and these results will be forthcoming in the near future. ${ }^{9}$ In addition, the procedural diversity shown in this report as well as the low rates of adverse events necessitate the development of a risk-adjustment methodology in IMproving Paediatric and Adult Congenital Treatment to allow for equitable comparisons among institutions in the future, work that is presently being completed within a focussed working group of the IMproving Paediatric and Adult Congenital Treatment registry.

Furthermore, one of the few case types that allowed comparison with recent data available from other registries was endomyocardial biopsy, which accounted for $>40 \%$ of all "non-core" cases submitted to the IMproving Paediatric and Adult Congenital Treatment registry. Among the biopsy cases submitted to the IMproving Paediatric and Adult Congenital Treatment registry, the rate of any adverse event was $3.2 \%$ and major adverse events $0.6 \%$. This is very similar to recent results reported from the Congenital Cardiac Catheterization Project on Outcomes registry collected from eight centres, which documented an overall adverse event rate of $3.3 \%$ with $1.1 \%$ of high-severity adverse events. ${ }^{16}$ Median case times were 38 minutes for the biopsy data derived from the IMproving Paediatric and Adult Congenital Treatment registry and 30 minutes for the Congenital Cardiac Catheterization Project on Outcomes data.

Pulmonary artery rehabilitation, both proximal and peripheral, accounted for $>10 \%$ of all "non-core" cases. Individual data were difficult to compare with other series, as most reports were single-centre experiences, with only the Congenital Cardiac Catheterization Project on Outcomes registry reporting a recent multi-centre experience. ${ }^{7}$ Furthermore, the location of the intervention, proximal versus peripheral, as well as the number of interventions performed make it difficult to compare these data with other reports. Within the IMproving Paediatric and Adult Congenital Treatment data set, the incidence of any adverse event associated with pulmonary artery balloon angioplasty was $13.0 \%$ for proximal locations and $13.8 \%$ for peripheral locations, with major adverse events occurring in $<1 \%$ of the cases. In contrast, the Congenital Cardiac Catheterization Project on Outcomes registry reported rates of high severity adverse events (level 3-5) for different types of balloon angioplasty between 9 and $14 \% ;^{7}$ however, it is important to point out that level 3-5 adverse events as reported in the Congenital Cardiac Catheterization Project on Outcomes registry do include many adverse events that would not be classified as a major adverse event in the IMproving Paediatric and Adult Congenital Treatment registry, and therefore comparison with life-threatening adverse events - only level 4 and level 5 - of the Congenital Cardiac Catheterization Project on Outcomes registry would be more appropriate. $^{14}$

\section{Limitations}

Despite being a large study cohort including a total of 8021 cases, the number of cases within individual case types was frequently still small, thereby somewhat limiting the ability to draw any more stringent conclusions from the data presented for many of the case categories. Furthermore, outcome metrics, other than adverse events, have not been firmly established for any of the presented case types, and as such these data do not 
allow for any conclusions relating to procedural efficacy; however, providing these data is crucial to aid establishing those metrics in the future. In addition, although attempts were made to present well-defined case types, the IMproving Paediatric and Adult Congenital Treatment registry does not collect some other important details such as whether multiple interventions of the same case category were performed in the same case in a given patient - for example, balloon angioplasty in a peripheral pulmonary artery branch in a single lesion would be categorised as the same case category in a case where five peripheral branches were treated through balloon angioplasty, which naturally would have an important impact on parameters such as, for example, case time. Other limitations of this study are inherent to the IMproving Paediatric and Adult Congenital Treatment registry itself, which includes, for example, the lack of longitudinal data collection. Comparison of adverse event data with other registries is often limited by very different definitions and inclusions of adverse events into the specific registries.

\section{Conclusions}

The IMproving Paediatric and Adult Congenital Treatment registry has provided important data on the frequency and spectrum of cardiac catheterisation procedures performed in the present era. For many procedures, more data and work are needed to identify more subtle differences between case categories, especially as it relates to the incidence of major adverse events, and to further develop a risk-adjustment methodology to allow equitable comparisons among institutions.

\section{Acknowledgements}

None.

\section{Financial Support}

This research received no specific grant from any funding agency, commercial, or not-for-profit sectors.

\section{Conflicts of Interest}

None.

\section{References}

1. Bergersen L, Marshall A, Gauvreau K, et al. Adverse event rates in congenital cardiac catheterization - a multi-center experience. Catheter Cardiovasc Interv 2010; 75: 389-400.

2. Everett AD, Ringel R, Rhodes JF, et al. Development of the MAGIC congenital heart disease catheterization database for interventional outcome studies. J Interv Cardiol 2006; 19: 173-177.

3. Jenkins KJ, Beekman Iii RH, Bergersen LJ, et al. Databases for assessing the outcomes of the treatment of patients with congenital and paediatric cardiac disease - the perspective of cardiology. Cardiol Young 2008; 18 (Suppl 2): 116-123.

4. Holzer R, Qureshi S, Ghasemi A, et al. Stenting of aortic coarctation: acute, intermediate, and long-term results of a prospective multiinstitutional registry - Congenital Cardiovascular Interventional Study Consortium (CCISC). Catheter Cardiovasc Interv 2010; 76: 553-563.

5. Holzer R, Qureshi S, Ghasemi A, et al. Stenting of aortic coarctation: acute, intermediate, and long-term results of a prospective multiinstitutional registry - Congenital Cardiovascular Interventional Study Consortium (CCISC). Catheter Cardiovasc Interv 2010; 76: 553-563.

6. Everett $\mathrm{AD}$, Jennings J, Sibinga $\mathrm{E}$, et al. Community use of the amplatzer atrial septal defect occluder: results of the multicenter MAGIC atrial septal defect study. Pediatr Cardiol 2009; 30: 240-247.

7. Holzer RJ, Gauvreau K, Kreutzer J, et al. Balloon angioplasty and stenting of branch pulmonary arterues: adverse events and procedural characteristics - results of a multi-institutional registry. Circ Interv 2011; 4: 287-296.

8. Holzer RJ, Gauvreau K, Kreutzer J, Hirsch R, Torres A, Bergersen L. Safety and efficacy of balloon pulmonary valvuloplasty: a multicenter experience. JACC 2012; 80: 663-672.

9. Bergersen L, Gauvreau K, Foerster SR, et al. Catheterization for Congenital Heart Disease Adjustment for Risk Method (CHARM). JACC Interv 2011; 4: 1037-1046.

10. Moore JW, Vincent RN, Beekman RH 3rd, et al. Procedural results and safety of common interventional procedures in congenital heart disease: initial report from the national cardiovascular data registry. J Am Coll Cardiol 2014; 64: 2439-2451.

11. Martin GR, Beekman RH, Ing FF, et al. The IMPACT registry: IMproving pediatric and adult congenital treatments. Semin Thorac Cardiovasc Surg Pediatr Card Surg Annu 2010; 13: 20-25.

12. Messenger JC, Ho KK, Young CH, et al. The National Cardiovascular Data Registry (NCDR) Data Quality Brief: the NCDR Data Quality Program in 2012. J Am Coll Cardiol 2012; 60: 1484-1488.

13. Vincent RN, Moore J, Beekman RH, et al. Procedural characteristics and adverse events in diagnostic and interventional catheterizations in pediatric and congenital heart disease: initial report from the IMPACT Registry. Cardiol Young 2015, Accepted for publication.

14. Lin $\mathrm{CH}$, Hegde S, Marshall AC, et al. Incidence and management of life-threatening adverse events during cardiac catheterization for congenital heart disease. Pediatr Cardiol 2014; 35: 140-148.

15. Bergersen L, Gauvreau K, Marshall A, et al. Procedure-type risk categories for pediatric and congenital cardiac catheterization. Circ Cardiovasc Interv 2011; 4: 188-194.

16. Daly KP, Marshall AC, Vincent JA, et al. Endomyocardial biopsy and selective coronary angiography are low-risk procedures in pediatric heart transplant recipients: results of a multicenter experience. J Heart Lung Transplant 2012; 31: 398-409. 